\title{
Transition-to-practice guidelines: Enhancing the quality of nursing education
}

\author{
T Bvumbwe, ${ }^{1} \mathrm{PhD} ; \mathbf{N}$ Mtshali, ${ }^{2} \mathrm{PhD}$
}

${ }^{1}$ Department of Nursing and Midwifery, Faculty of Health Sciences, Mzuzu University, Luwinga, Malawi

${ }^{2}$ School of Nursing and Public Health, University of KwaZulu-Natal, Durban, South Africa

Corresponding author: T Bvumbwe (bvumbwe.tm@mzuni.ac.mw)

\begin{abstract}
Background. A de-link between initial training and transition to practice has been reported. Effective transitioning to practice enhances competences and confidence among newly graduated nurses.

Objectives. To develop transition-to-practice guidelines as a tool to complement efforts to improve nursing education in Malawi.

Methods. A multi-method design was used within the framework of the Stufflebeam context, input, process, product (CIPP) model. Analysis of reports from a nursing conference derived four core concepts, highlighting the context within which goals for transition-to-practice guidelines needed to be focused on. A panel discussion suggested guidelines based on these concepts. Review meetings and a review of the literature, local policies and standards were conducted to provide input to enhance credibility and reproducibility of the proposed guidelines. Consensus workshops involving nurse educators, nursing clinical preceptors, nurse practitioners and policymakers were conducted as a process evaluation for the guidelines.

Results. Four core concepts emerged from the process of guideline development. Eleven guideline statements were formulated as a product of the guideline development process. Although newly graduated nurses are exposed to various clinical settings during college training, nurses' skills and clinical judgement are still rather weak and need more formal support. The guidelines provide assistance for transition to practice among newly graduated nurses.

Conclusion. Nursing education is a complex process that starts at student recruitment and should effectively progress until transition to practice Transition-to-practice guidelines to complement other guidelines in nursing education are timely in Malawi.
\end{abstract}

Afr J Health Professions Educ 2018;10(1):66-71. DOI:10.7196/AJHPE.2018.v10i1.898

There is global consensus that the performance of a healthcare system largely depends on a competent nursing and midwifery workforce. Nurses form the backbone of the healthcare system and are a universal access point for almost $80 \%$ of healthcare users, especially in primary healthcare settings. However, nurses continue to face challenges in the 21 st century, which are more complex and have changed healthcare delivery, especially in poorresource settings.

Globally, there is an increased demand for an efficient and effective nursing workforce. Evidence shows that, despite the increasing complexity of nursing practice, there is a wide gap between theory and practice. ${ }^{[1]}$ Clinical education remains central to the nursing curriculum and forms the foundation for bridging the gap between theory and practice. Clinical education prepares nurses for skills and competences to effectively provide safe, quality care in complex settings. ${ }^{[2]}$ In Malawi, there are reports of poor competences and negative attitudes of nurses towards patients and nursing care. Therefore, nursing education falls short of the expectation to produce sufficient and well-trained nurses. ${ }^{[3]}$ Missen et al..$^{[4]}$ reported that inadequacy in the preparation of nurses for practice is a worldwide problem. A de-link between initial training and transition to practice has been reported in many setting. ${ }^{[5]}$

Effective transition to practice enhances socialisation, and improves competences $^{[6]}$ and confidence among nurses. Spector et al. ${ }^{[7]}$ indicated that hospitals that use established transition programmes reported higher retention rates. Nurses also reported fewer patient errors, employed fewer negative safety practices, and had lower stress levels and better job satisfaction. It is against this background that we developed transition-topractice guidelines in nursing.

\section{Assumption underlying the guidelines}

The transition-to-practice guidelines are built on the assumption that training of nurses is a complex process. Its outcome is based on an interactive relationship between academic theory and practice, which is well regulated at all stages. The task to narrow the theory-practice gap requires a co-ordinated process between academic and practice settings, which strengthens the ability of newly graduated nurses to perfect what they have learnt in training institutions in complex practice settings.

\section{Purpose of the guidelines}

Transition to practice of new graduates forms a critical component of the overall nursing education process. Well-developed transition-to-practice programmes ensure a competent nursing workforce that is adequately prepared to deliver within a complex healthcare system. Transition guidelines will help to increase nurses' confidence and competences, increase patient safety and improve overall quality of nursing care in Malawi. The guidelines will support formal programmes that are designed to assist progression of newly graduated nurses from training to practice. The purpose of this work was therefore to develop guidelines for transition to practice as a tool to complement nursing education in Malawi. 


\section{Guidelines stakeholders}

The training of nurses is a complex process that starts at recruitment into a nursing programme and progresses to continued professional development throughout their working life. This complex process overlaps with various environments and various stakeholders. The latter are individuals who take a participatory role in the training of nurses and include the student, nurse educator, regulatory body and healthcare institution.

\section{Nursing educator}

Nurse educators align nursing curricula to the practising needs of the nursing students. The nurse educator imparts knowledge to nursing students, who use it to perfect their practising skills and competences.

\section{Regulatory body}

The regulatory body stipulates various scopes of practice and expected competency levels that a newly graduated nurse should achieve to be certified as safe and competent to practise. This body regulates nurse education and practice, and both have to be aligned in the preparation of the new nurse.

\section{Healthcare institution}

Healthcare institutions have a unique culture in which the new graduate has to adapt to ensure efficiency and effectiveness in delivery of quality services. The nurse manager's task is to ensure that new graduates increase their performance to achieve health goals for healthcare users.

\section{Newly graduated nurses}

A newly graduated nurse is a key stakeholder at the centre of an interactional relationship among healthcare institution, regulatory body and nurse educator.

\section{Guidelines development process}

A multi-method design was used for the development of the transition-to-practice guidelines (Fig. 1). Initially, reports from a national nursing education research conference in 2015 were analysed using thematic analysis to propose core concepts. Table 1 summarises research reports that were presented during the conference. Four core concepts were derived from analysis of the reports. On the last day of the conference, 8 nursing experts were invited

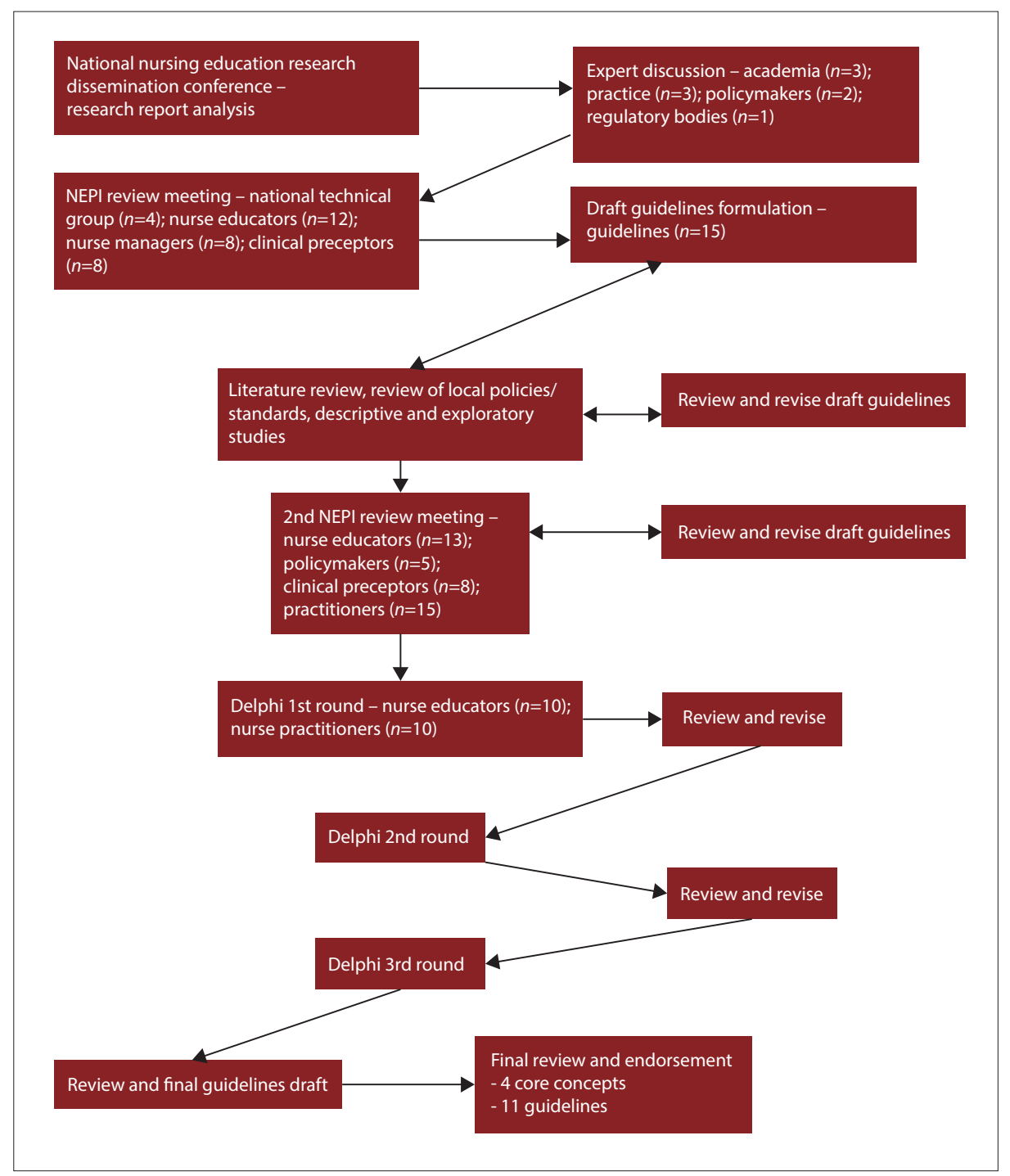

Fig. 1. Process of guidelines development for transition to practice. (NEPI = Nursing Education Partnership Initiative.)

to a panel discussion based on their specific expertise in nursing education and clinical practice to discuss the reports. Table 2 presents demographic characteristics of participants during the expert discussion. The discussion was open and guided by two questions followed by probes: 'What is your comment about the quality of nursing education in Malawi based on the research reports presented during the conference?' and 'What recommendations would you give to improve nursing education?'. Context evaluation by means of the panel discussion determined goals that would be addressed by the transition-to-practice guidelines. Context evaluation described the current state of the nursing education gap.

A Nursing Education Partnership Initiative (NEPI) project gathered members of the NEPI technical working group $(n=4)$, nurse educators $(n=12)$, nursing managers $(n=8)$ and nursing clinical preceptors $(n=8)$ from teaching hospitals to review strategies being implemented to improve nursing education in Malawi in view of the outcome of the expert discussion. The panel of experts and those at the review meetings discussed the core concepts derived from the nursing education conference. Key points were summarised and draft transition-to-practice guidelines in nursing education were formulated.

The draft guidelines then guided a review of local policies and nursing education standards. A review of the literature and a descriptive exploratory study were conducted to contribute towards an input evaluation to enhance credibility and reproducibility of the proposed guidelines. The guidelines were then discussed 
Table 1. Summary of research reports for the nursing education research conference

\begin{tabular}{ll}
\hline Core concepts & Research report \\
\hline Academic clinical & Where is the grade coming from? Problems and challenges \\
collaboration & in evaluating the clinical performance of nursing students
\end{tabular}

Key findings

collaboration

Evaluation of students' clinical performance is a vital component of nursing education; it should be conducted in a manner that effectively determines students' clinical proficiency. Students become preoccupied with building relationships with clinical nurses to obtain good grades

Registered nurses' experiences with the clinical teaching environment in Malawi

Clinical teaching and learning inadequately prepares students for practice owing to challenges of inadequate faculty support, poor clinical learning environment, poor competence among nurses and unsupportive working conditions

Can research improve nursing and midwifery education in Malawi? (Keynote address)

Evidenced-

based practice strengthening

Patient-centred care in nursing and midwifery education

Theory-practice gap reduction

Innovations in nursing training
Strategies for the implementation of clinical practice guidelines in intensive care: a systematic review Involvement of registered nurses in clinical teaching of nursing students in central hospitals in Malawi

Assessing quality of the clinical learning environment for nursing and midwifery students in northern Malawi

\section{Clinical teaching in clinical situations}

An investigation of stressors among Malawian nursing and midwifery students

Enhancing students' moral competence in practice: challenges experienced by Malawian nurse teachers

Exploring knowledge and perceptions of tutors of the use of a problem-based learning approach in Christian Health Association of Malawi nursing colleges

Factors affecting clinical performance of nursing and midwifery technician students at three nursing colleges in southern Malawi

Teaching and learning methodology in nurse/midwife education

Knowledge and attitudes of nursing and midwifery learners and educators towards self-directed learning in Malawi
Evidence-based practice requires that both nurse educators and nurse practitioners engage in collaborative research. Research builds on knowledge for nurses

Quality of nursing care improves when care is based on objective assessment of patients' needs. Nursing education emphasises evidencebased provision of nursing care

Practice guidelines strengthen provision of quality care to the patient in the intensive care unit

Registered nurses possess adequate experience regarding practice. Their involvement increases changes for narrowing a theory-practice gap that exists owing to lack of integration between what students learn in class and what happens in practice settings

The nature of the clinical learning environment has a direct impact on the achievements of the clinical learning outcomes. However, the clinical learning environment is characterised by a lack of resources, poor faculty support, and a lack of collaboration between academia and practice in training students

Students learn better in clinical situations that provide adequate support from clinical personnel

Clinical learning is stressful for students owing to the nature of the clinical learning environment, especially for newer students. With time, students adapt to the challenges of the clinical environment

A less authoritarian learning climate may enhance critical reflection and discussion between students, teachers and nurses. Students develop moral competence when they are given an opportunity to reflect

Nurse educators need capacity building in teaching approaches to promote achievement of learning outcomes among students

Students' clinical performance is affected by a poor clinical learning environment. Nurses' attitude towards students, availability of faculty support during clinical practice and lack of resources are important aspects of a clinical learning environment

Use of various methods in teaching enhances acquisition of knowledge and skills by nurses. Clinical mentorship increases the chance for students to learn during practice

Adequate orientation of students towards teaching approaches increases their positive attitude towards these approaches and reviewed in a consensus workshop. Nurse educators ( $n=13)$, nursing clinical preceptors $(n=8)$, nurse practitioners $(n=15)$ and policymakers $(n=5)$ participated in the workshop to provide information that can be used to guide the implementation of the guidelines, procedures and activities, as well as being a means to identify successes and failures.

Consensus procedure followed the reviews and involved three rounds of Delphi stages. First, Delphi involved nurse educators $(n=10)$ and nurse practitioners $(n=10)$ to explore their opinions on the guidelines. Results from the first round of the discussion informed a questionnaire for the last two Delphi stages. The researcher (TB) reviewed and produced a final draft of the guidelines, which was presented to an education committee for approval as the final product of the guideline-development process. Table 3 presents final transition-to-practice guidelines that were developed by means of the abovementioned process. 


\begin{tabular}{|c|c|c|c|c|}
\hline Characteristics & Nurse educators & Nurse practitioners & Policymakers & Regulatory bodies \\
\hline \multicolumn{5}{|l|}{ Gender } \\
\hline Male & 1 & - & - & - \\
\hline Female & 2 & 3 & 2 & 1 \\
\hline \multicolumn{5}{|l|}{ Age, years } \\
\hline$\leq 30$ & - & - & - & - \\
\hline $31-40$ & 1 & 2 & - & - \\
\hline$\geq 41$ & 2 & 1 & 2 & 1 \\
\hline \multicolumn{5}{|c|}{ Education qualification } \\
\hline Bachelor & - & 1 & - & - \\
\hline Master & 3 & 2 & 2 & 1 \\
\hline $\mathrm{PhD}$ & - & - & - & - \\
\hline \multicolumn{5}{|c|}{ Length of service, years } \\
\hline$\leq 5$ & - & - & - & - \\
\hline $6-15$ & 1 & - & - & - \\
\hline$\geq 16$ & 2 & 3 & 2 & 1 \\
\hline \multicolumn{5}{|l|}{ Publications, $n$} \\
\hline 0 & - & 1 & - & - \\
\hline$\leq 2$ & 1 & 2 & 1 & 1 \\
\hline$\geq 3$ & 2 & - & 1 & - \\
\hline
\end{tabular}

Table 3. Guidelines for transition to practice in nursing education

Core concept 1: Academic clinical collaboration

Transition-to-practice programmes should show evidence of collaboration between training and practice institutions

Transition-to-practice programmes should be implemented in liaison between co-ordinators of the transition programme from both training and practice institutions

\section{Core concept 2: Evidence-based practice strengthening}

Academic and practice settings should mutually engage to develop innovative ways to support transition of newly graduated nurses

Newly graduated nurses should undergo a formal performance appraisal by responsible mentors from the hosting practice institution and nurses' training or linked college

\section{Core concept 3: Theory-practice gap reduction}

A formal transition-to-practice programme that meets the expected learning outcomes of new graduates should be approved by the Nurses and Midwives Council of Malawi

All students entering the transition-to-practice programme should show evidence of fulfilling minimum requirements for completion of the nursing training programme

Transition to practice should be done at health institutions that meet the minimum set standards for clinical training placement

Newly graduated nurses should be engaged within the first 6 months after graduation, as this is considered a critical period that needs transition support for nurses to consolidate what they learnt while in college

\section{Core concept 4: Innovation in nursing training approaches}

All registered nurses should be trained as clinical preceptors to support clinical mentorship of newly graduated nurses Transition to practice should be done under supervision of a trained preceptor

Transition to practice is considered as a period for orientation, preceptorship and specific professional development that allows personal and professional growth

\section{Guidelines development process: Outcome and discussion}

The national nursing education research conference comprised 18 research reports and 1 keynote speech. Before the conference, abstracts were independently assessed for inclusion by a Norwegian Church Aid conference committee. Four core concepts within the context evaluation of the research reports emerged and included academic and practice collaboration, evidence-based practice strengthening, theory-practice gap reduction and innovations in the approach to the training of nurses, derived from analysis of the reports. Draft guidelines for transition to practice were developed from these four core concepts.

\section{Core concept 1: Academic and practice collaboration}

The nursing curriculum needs to be aligned to the clinical setting to ensure 
that graduates are equipped to face the challenges of a complex and dynamic healthcare delivery system. Greenwood ${ }^{[8]}$ argued that the effectiveness of qualified nursing graduates should become the responsibility of both the training institutions and clinical practice. Expert review revealed that preparing a sustainable, competent nursing and midwifery workforce is a shared responsibility between academia and the practice setting. Some participants highlighted the following:

'... a nurse will never be fully produced by training institutions without practice institutions.' (AC, Nurse educator)

'Training for practice requires that those trained should practise within the practice settings that embrace theory and practice integration.' (TL, Nurse regulator)

Academic-practice collaboration is an important mechanism for strengthening nursing education, practice and research. Despite the increasing effort to bridge the theory-practice gap, the lack of formal partnerships between academia and practice leads to disintegrated efforts in the improvement of nursing education in Malawi. Data collected from consensus meetings indicated that the guidelines that were developed put in place measures to ensure that academic and practice settings work collaboratively towards a competent and efficient nursing graduate (Table 1). There is hope for an improved nursing education system in Malawi if nurse educators and practitioners understand and appreciate the academicpractice partnership, its benefits, elements and challenges.

Lack of a transition-practice programme exposes new nurses to the loss of the nursing education support system. During guideline review meetings, participants highlighted the importance of maintaining college support during the transition period. Duchscher ${ }^{[9]}$ reported that if new nurses do not have immediate access to previous educators to provide intellectual counsel, emotional support, practice consultation and feedback, feelings of isolation and self-doubt increase. Access to support from peers and colleagues is reported to be an important link for nurses' development. A participant indicated that:

'... the mentorship programme will expose newly graduated nurses to an environment with appropriate support from experienced nurses.' (GC, Policymaker)

Nurse educators could take a leading role in designing curricula for transition programmes.

\section{Core concept 2: Evidence-based practice strengthening}

Evidence-based nursing promotes the use of contemporaneous recent research findings as the basis for clinical decisions. ${ }^{[10]}$ The newly graduated nurse must develop cognitive and emotional knowledge and technical skills and be able to apply this knowledge in practice. This will help nurses to make well-grounded decisions and deliver evidence-based nursing. Findings of this study highlight that new nurses must be able to synthesise evidence-based information with critical thinking skills. Transition-topractice programmes should support an understanding of the importance of quality care and of their role in the continuum of care among new nurses. For quality care provision, newly graduated nurses have to develop the clinical judgement that experienced nurses possess:

'... the process of transition exposes the newly graduated nurses to practice culture that the newly graduated gets socialised into.' (ET, Nurse educator)
Opinions from consensus discussions showed that newly graduated nurses discover the practical knowledge that is necessary for clinical judgement as they undergo transition programmes. Expert discussion revealed that clinical decisions should be evidence based and that an understanding of the knowledge sources that newly graduated nurses use is important to safeguard quality of care. Voldbjerg et al. ${ }^{[11]}$ argued that, during the transition phase, feelings of confidence and ability to use critical thinking and reflection have a great impact on knowledge sources incorporated in clinical decisions.

\section{Core concept 3: Theory-practice gap reduction}

The literature shows a mismatch between nursing theory and practice. ${ }^{[12]}$ Differing perspectives exist between nurses in the practice sector and those in the education sector with regard to the practice readiness of new graduates. Wolff et al. ${ }^{[13]}$ recommended a shift in the discourse around practice readiness, whereby nurses from all sectors should focus on unique, innovative and co-operative solutions to ensure the effective transition of all nursing graduates in the 21 st century healthcare system. Wide gaps in theory and practice among new nurses are being reported. ${ }^{[14]}$ Consensus discussion pointed out that transition-to-practice programmes help to reduce the gap between what students learn in class and what is expected of them in practice:

'Newly graduated nurses need a period of orientation to real practice. This helps them integrate into practice what they learn during training.' (TN, Nurse practitioner)

\section{Core concept 4: Innovations in the training of nurses}

There was strong argument from the consensus discussions that initial experiences of graduates can shape the development of nurses during their careers. Findings of the study indicated the critical importance of welcoming the new nurse into an inquisitive, supportive environment, where good staff relationships flourished. This is consistent with Spector et al.s ${ }^{[7]}$ report, which highlighted that transition-to-practice programmes that included patient-centred care, communication and networking, quality improvement, evidence-based practice, informatics, safety, clinical reasoning, feedback, reflections and specialty knowledge in an area of practice provided better support for newly graduated nurses.

Data from a panel of experts indicated that preceptorship proved to be an essential and possible way to start as a new nurse. There was a general understanding in the consensus meetings that preceptors provide a supportive environment for new nurses to develop their confidence as independent professionals and refine their skills, values and behaviours. Matua et al. ${ }^{[15]}$ noted that having expert support and learning from best practice give new nurses a foundation for lifelong learning through professional feedback. Studies showed that supportive behaviour and constructive feedback from qualified nurses and involvement of newly graduated nurses in all aspects of decision-making during care provision are important features that assist in developing the new nurse. Newly qualified nurses become well aware of their inexperience. General feedback from the data showed that the guidelines therefore highlight the need for effective preparation of preceptors to fill this important role and help new graduates develop their skills and competences:

'... registered nurses need to be equipped with knowledge and skills to take newly graduated nurses through a transition process that allows innovation and creativity. (GC, Policymaker)' 


\section{Conclusion and implications for nursing education}

Nursing education prepares a sufficient number of highly qualified nurses, who are necessary for the complex healthcare demands. A key concern of nurse educators is preparing graduates for practice. Findings of our study showed that transition to practice has not been adequately addressed in Malawi. New nurses require adequate support because they face a new environment, new expectations and new roles when they join a practice. These guidelines offer a direction on how transition to practice could be conducted in Malawi to ensure effective preparation of newly graduated nurses for practice. The guidelines complement the existing standards in guiding nursing education practice. Proper transition-to-practice programmes are significant for new graduates' professional growth.

\section{Study limitation}

There is one limitation to this study that needs to be addressed. Malawi plans to introduce an internship programme for nursing training programmes. These guidelines may serve the same purpose. Consensus should be reached to have either a transition-to-practice programme or an internship programme. One general name needs to be adopted for the programme.

Acknowledgements. Thanks to all nurses who participated in the study. Author contributions. TB conceptualised the study and collected the data. TB and NM analaysed the data and drafted and approved the final manuscript.
Funding. The study was supported by ICAP through a NEPI. Conflicts of interest. None.

1. Ajani K, Moez S. Third World Conference on Educational Sciences - 2011 gap between knowledge and practice in nursing. Procedia Soc Behav Sci 2011;15:3927-3931. https://doi.org/10.1016/j.sbspro.2011.04.396

2. Wells L, McLoughlin M. Fitness to practice and feedback to students: A literature review. Nurse Educ Pract 2014;14(2):137-141. https://doi.org/10.1016/j.nepr.2013.08.006

3. Msiska G, Smith P, Fawcett T. The 'lifeworld' of Malawian undergraduate student nurses: The challenge of learning in resource poor clinical settings. Int J Afr Nurs Sci 2014;1:35-42. https://doi.org/10.1016/jijans.2014.06.003

4. Missen K, McKenna L, Beauchamp A. Work readiness of nursing graduates: Current perspectives of graduate nurse 4. Missen K, McKenna L, Beauchamp A. Work readiness of nursing graduates: Current perspectives of gradua
program coordinators. Contemp Nurse 2015;51(1):27-38. https:///oi.org/10.1080/10376178.2015.1095054

program coordinators. Contemp Nurse $2015 ; 51(1): 27-38$. https://doi.org/ $10.1080 / 10376178.2015 .1095054$
5. Hofler L, Thomas K. Transition of new graduate nurses to the workforce challenges and solutions in the changing Hofler L, Thomas K. Transition of new graduate nurses to the workforce challenges and solutions in the ch
health care environment. North Carolina Med J 2016;77(2):133-136. https://doi.org/10.18043/ncm.77.2.133

health care environment. North Carolina Med J 2016;77(2):133-136. https://doi.org/10.18043/ncm.77.2.133
6. Komaratat S, Oumtanee A. Using a mentorship model to prepare newly-graduated nurses for competency. J Contin Educ Nurs 2009;40(10):475-480. https://doi.org/10.3928/00220124-20090923-02

7. Spector N, Blegen M, Silvestre J, et al. Transition-to-practice study in hospital settings. J Nurs Educ 2012;5(4):24-38 https://doi.org/10.1016/s2155-8256(15)30031-4

8. Greenwood J. Critique of the graduate nurse: An international perspective. Nurse Educ Today 2000;20(1):17-23. https://doi.org/10.1054/nedt.2000.0424

9. Duchscher JE. Transition shock: The initial stage of role adaptation for newly-graduated registered nurses. I Adv Nurs 2009;65(5):1103-1113. https://doi.org/10.1111/j.1365-2648.2008.04898.x

10. Rosenberg W, Donald A. Evidence based medicine: An approach to clinical problem-solving. BMJ 1995;310(6987):1122-1126. https://doi.org/10.1136/bmj.310.6987.1122

11. Voldbjerg SL, Gronkjaer M, Sorensen EE, Hall EO. Newly-graduated nurses' use of knowledge sources: A metaethnography. J Adv Nurs 2016;72(8):1751-1765. https://doi.org/10.1111/jan.12914

12. Rich KL, Nugent KE. A United States perspective on the challenges in nursing education. Nurse Educ Today 2010:30(3):228-232. A United States perspective on the c15

13. Wolff AC, Pesut B, Regan S. New graduate nurse practice readiness: Perspectives on the context shaping our understanding and expectations. Nurse Educ Today 2010;30(2):187-191. https://doi.org/10.1016/j.nedt.2009.07.01 14. Monaghan T. A critical analysis of the literature and theoretical perspectives on theory and practice gap amongst newly-qualified nurses within the United Kingdom. Nurse Educ Today 2015;35(8):e1-e7. https://doi. org/10.1016/j.nedt.2015.03.006

15. Matua G, Seshan V, Akintola A, Thanka A. Strategies for providing effective feedback during preceptorship: Perspectives from an Omani Hospital. J Nurs Educ Pract 2014;4(10):24-31. https://doi.org/10.5430/jnep.v4n10p24

Accepted 14 September 2017. 